\title{
Non-Markovian Dynamics of Quantum and Classical Correlations in the Presence of System-Bath Coherence
}

\author{
Chuan-Feng Li*, Hao-Tian Wang, Hong-Yuan Yuan, Rong-Chun Ge, and Guang-Can Guo \\ Key Laboratory of Quantum Information, \\ University of Science and Technology of China, \\ CAS, Hefei, 230026, People's Republic of China
}

(Dated: October 12, 2018)

\begin{abstract}
We present a detailed study of the dynamics of correlations in non-Markovian environments, applying the hierarchy equations approach. This theoretical treatment is able to take the system-bath interaction into consideration carefully. It is shown that crosses and sudden changes of classical and quantum correlations can happen if we gradually reduce the strength of the interactions between qubits. For some special initial states, sudden transitions between classical and quantum correlations even occur.
\end{abstract}

PACS numbers: 03.65.Ta, 03.65.Yz

\footnotetext{
* email: cfli@ustc.edu.cn
} 


\section{INTRODUCTION}

In the field of quantum information and computation, a great amount of meaningful investigations have been done to explore the dynamics of two-level systems in environments with different properties [1-4]. Information extracted from a bipartite system comes to the center of our interests because in this simplest scenario we can not only conveniently introduce our theoretical calculations but also obtain the same results even with different definitions. In a two-qubit system, quantum mutual information has been defined as the largest amount of information which we can extract between the qubits [5]. This mutual information consists of two different parts, the classical correlation and the quantum correlation. Quantum correlation, which is equivalent to quantum discord in some special cases [6], can also be divided as quantum entanglement and non-entanglement quantum correlation [7]. Many works have been focused on the different dynamics between quantum correlation and entanglement [1].

The most popular definitions of quantum information are discussed by Vedral, in which the total correlations (quantum mutual information) comes to the form as [8]

$$
\mathcal{I}\left(\rho_{A B}\right)=S\left(\rho_{A}\right)+S\left(\rho_{B}\right)-S\left(\rho_{A B}\right)
$$

where $\rho_{A(B)}$ is the reduced density matrix of $\rho_{A B}$ and $S(\rho)$ represents the von Neumann entropy which can be calculated as $S(\rho)=-\Sigma_{i} \lambda_{i} \log _{2} \lambda_{i}$, in which $\lambda_{i}$ is the eigenvalue of $\rho$. Classical correlation can be obtained from the measurements of subsystem $B$ under the complete set of orthonormal projectors in the form

$$
\Pi_{\|}=\sin ^{2} \theta|1\rangle\left\langle 1\left|+\cos ^{2} \theta\right| 0\right\rangle\langle 0|+\sin \theta \cos \theta\left(e^{i \phi}|1\rangle\left\langle 0\left|+e^{-i \phi}\right| 0\right\rangle\langle 1|\right)
$$

and

$$
\Pi_{\perp}=\cos ^{2} \theta|1\rangle\left\langle 1\left|+\sin ^{2} \theta\right| 0\right\rangle\langle 0|-\sin \theta \cos \theta\left(e^{i \phi}|1\rangle\left\langle 0\left|+e^{-i \phi}\right| 0\right\rangle\langle 1|\right),
$$

where $|1\rangle$ and $|0\rangle$ are the excited and ground states of the qubit. Then classical correlation can be expressed as [2, 9]

$$
\mathcal{C}\left(\rho_{A B}\right)=\max _{\theta, \phi}\left[S\left(\rho_{A}\right)-\Sigma_{j} q_{j} S\left(\rho_{A}^{j}\right)\right]
$$

with $\rho_{A}^{j}=\operatorname{Tr}_{B}\left[\left(\mathbb{I} \otimes \Pi_{j}\right) \rho_{A B}\left(\mathbb{I} \otimes \Pi_{j}\right)\right] / q_{j}, q_{j}=\operatorname{Tr}_{A B}\left[\rho_{A B}\left(\mathbb{I} \otimes \Pi_{j}\right)\right]$, and $j=\|, \perp$. Since the total correlations can also be expressed as

$$
\mathcal{I}\left(\rho_{A B}\right)=\mathcal{C}\left(\rho_{A B}\right)+\mathcal{Q}\left(\rho_{A B}\right)
$$


then the quantum correlation is

$$
\mathcal{Q}\left(\rho_{A B}\right)=\mathcal{I}\left(\rho_{A B}\right)-\mathcal{C}\left(\rho_{A B}\right)
$$

With above basic equations, the left work is to give the values of the system matrix $\rho_{A B}$. In most scenarios we may find that the classical and quantum correlation would have mediocre behaviors, such as the result that classical correlation is always lager than quantum correlation. However, Many meaningful theoretical and experimental works have been done recently to demonstrate that with some special channels and a class of initial states, the classical correlation and quantum correlation will both remain constant in different regimes, and there is a sudden transition from the classical to the quantum decoherence [10 12].

In this work we demonstrate that in some common cases we can also obtain crosses of classical and quantum correlations and correlation sudden changes. By applying the hierarchy equations approach [4, 13] we set up the model of a two interacting qubits system in independent non-Markovian environments, and we also carefully take into account the presence of system-bath coherence, which has been proved to dramatically influence the dynamics of the quantum system. As the strength of the interaction between the two qubits decreases, we can find the crosses and sudden changes gradually appear. When there are not interactions at all, the changes will be the sharpest.

\section{THE MODEL AND THEORY}

We present a newly developed theoretical method called hierarchy equations approach to handle the system dynamics [4, 13]. This method can conveniently treat the scenario in which there is a non-Markovian environment. We know that both the quantum system and the heat bath have characteristic frequencies. Most of theoretical treatments would suppose a fast bath to make the Born and ultrafast bath approximations valid [4]. However, in this hierarchy equations approach, a slow noise bath can be applied. This kind of environment will record part of the information from the system to the bath and then return some to the system.

We set $\hbar=1$ throughout this report. The environment is divided into two independent baths interacting with two interacting qubits. Then we write the standard system Hamilton 
as

$$
H_{S}=\varepsilon\left(a_{1}^{\dagger} a_{1}+a_{2}^{\dagger} a_{2}\right)+\zeta\left(a_{1}^{\dagger}+a_{1}\right)\left(a_{2}^{\dagger}+a_{2}\right)
$$

where $\varepsilon$ is the energy gap of the two-level system. The subscripts 1 and 2 represent the two qubits, $a^{\dagger}$ and $a$ are creation and annihilation operators. $\zeta$ stands for the strength of the interactions between two qubits, so we can conveniently reduce its value until zero to observe the changes of the correlations. The heat bath consists of a set of oscillators, with the form [3, 4, 14]

$$
H_{B+S B}=\sum_{j}\left(\frac{p_{j}^{2}}{2 m_{j}}+\frac{1}{2} m_{j} \omega_{j}^{2} x_{j}^{2}\right)-\sum_{m, j} C_{m j}\left(a_{m}^{\dagger}+a_{m}\right) x_{j},
$$

$x_{j}, p_{j}, m_{j}$ and $\omega_{j}$ are coordinate, momentum, mass and frequency of the $j$ th harmonic oscillator, respectively. Then $H_{B}$ presents the Hamilton of the bath and $H_{S B}$ is the interaction between system and environment. $C_{m j}$ stands for the coupling strength of the $j$ th oscillator to the $m$ th qubit. All of the system-bath coupling information can be found though the spectral density function $J(\omega)$ and in this work we model it as the Lorentzian cutoff

$$
J(\omega)=\frac{\omega \eta \gamma}{\omega^{2}+\gamma^{2}}
$$

$\eta$ represents the system-bath coupling strength, and $\gamma$ is the characteristic frequency of the heat bath. When the fast bath condition is fulfilled as $\hbar \gamma \gg \varepsilon$, we can choose the initial state in which the system and bath are independent. If $\hbar \gamma \sim \varepsilon$ it represents a non-Markovian environment and that is just our scenario. In the present work we choose $\varepsilon=1.5 \delta$ and $\gamma=4 \delta$, and here $\delta$ stands for an energy unit. With a Fourier transform of the spectral density function we have

$$
F(t)=\sum_{k=0}^{\infty} c_{k} e^{-\gamma_{k}|t|}
$$

where $\gamma_{k}$ is a Bosonic Matsubara frequency, with $\gamma_{0}=\gamma$ and $\gamma_{k}=2 \pi k / \beta$ when $k \geq 1$. The coefficients in front of the exponential function in Eq.10 have values as $c_{0}=\frac{\eta \gamma}{2}(-i+\cot \beta \gamma / 2)$ and $c_{k}=2 \eta \gamma_{0} \gamma_{k} / \beta\left(\gamma_{k}^{2}-\gamma_{0}^{2}\right)$ when $k \geq 1$. The equation about the dynamics of the system using hierarchy equations approach, witch has the following form [4] 


$$
\begin{aligned}
\frac{d \rho_{\mathbf{n}}(t)}{d t}= & -\left(i \mathcal{L}+\sum_{m=1}^{2} \sum_{k=0}^{K} n_{m k} \gamma_{k}\right) \rho_{\mathbf{n}}(t)-\sum_{m=1}^{2}\left(\left(\frac{1}{\beta \gamma_{0}}-i \frac{1}{2}\right) \eta-\sum_{k=0}^{K} \frac{c_{k}}{\gamma_{k}}\right)\left[V_{m},\left[V_{m}, \rho_{\mathbf{n}}(t)\right]\right] \\
& -i \sum_{m=1}^{2} \sum_{k=0}^{K} n_{m k}\left(c_{k} V_{m} \rho_{n_{m k}-1}(t)-c_{k}^{*} \rho_{n_{m k}-1}(t) V_{m}\right) \\
& -i \sum_{m=1}^{2} \sum_{k=0}^{K}\left[V_{m}, \rho_{n_{m k}+1}(t)\right]
\end{aligned}
$$

where $\mathcal{L}$ is the Liouvillian operator of the two-level system with $\mathcal{L} \rho=\left[H_{S}, \rho\right]$, and $V_{m}=$ $a_{m}^{\dagger}+a_{m}$ with $m=1,2$.

In Eq.11 $\rho_{\mathbf{n}}$ are called auxiliary density operators (ADOs) [3]. The subscript $\mathbf{n}$ is a multi-index which can be extended as $n_{m k}$, where $m$ represents the number of qubits in the system and the $k$ represents that of the Bosonic Matsubara frequencies. The notation $n_{m k} \pm 1$ refers to an increase and decrease of this index. Only when all the values of the multiindex are zero it stands for the physical system density operator. And if one of the notation $n_{m k} \pm 1$ has a value under zero or the sum of all the notations exceed a limit [13], the density operator is set to be zero. The values in the ADOs contain a deal of important information about the system-bath interaction, which has been proved to be able to dramatically change the dynamics of the system. With a carefully consideration of the system-bath coupling we find that it is a important factor which can greatly influence our results of the system evolution. Another advantage of this theoretical treatment is the convenience of applying the method into computer programs. It is quite easy to numerically calculate the dynamics of the system density matrix though a program. This hierarchy equations approach will be a useful treatment in scenarios when the interaction between the system and the environment is very strong.

\section{THE RESULT AND DISCUSSION}

In this work the environment is non-Markovian and much more common than the ones in Ref. [1, 12]. We set $\eta=0.3 \delta, \gamma=4 \delta$ and $\beta=2.5 / \delta$. The initial state of the two-qubit system is a Bell state with $|\psi\rangle=1 / \sqrt{2}(|10\rangle-|01\rangle)$. As the system Hamilton only has diagonal and anti-diagonal elements, the system density matrix will evolve into $X$ states [15], which can represent a great number of quantum systems. We use numerical method to calculate the mutual information, classical correlation and quantum correlation with enough precision. 


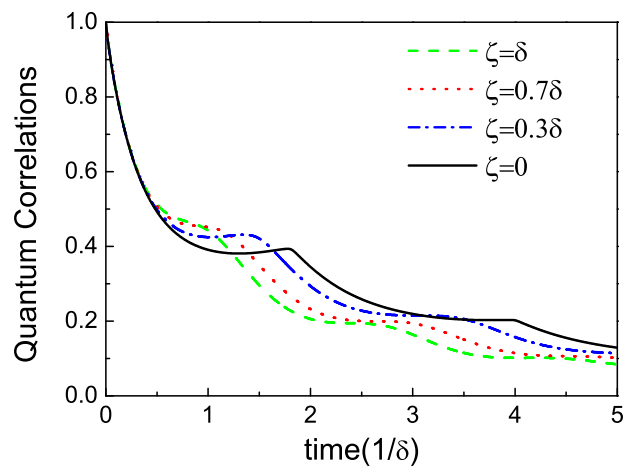

FIG. 1: (color online) The green dashed, red dotted, blue dash-dotted and black solid lines represent the quantum correlations as functions of time with $\zeta=\delta, 0.7 \delta, 0.3 \delta$ and zero, respectively.

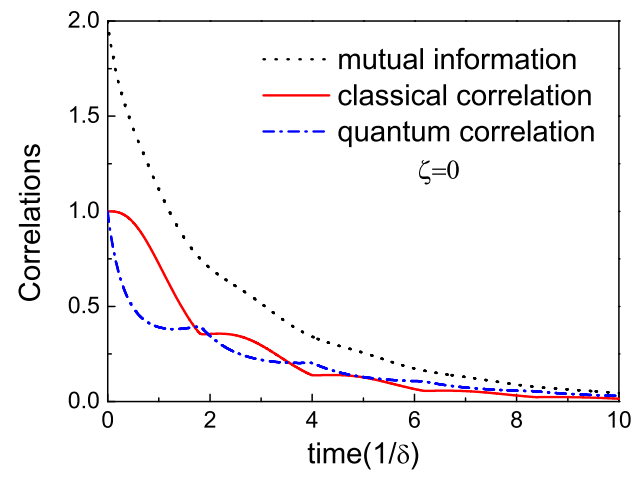

FIG. 2: (color online) The black dotted, red solid and blue dash-dotted lines represent the mutual information, classical correlation and quantum correlation as functions of time, respectively.

In order to show the correlation sudden changes, we set $\zeta=\delta, 0.7 \delta, 0.3 \delta$ and 0 respectively in Figure1. Obviously, with the decrease of $\zeta$, the quantum correlation becomes sharper and sharper. When $\zeta=0$, a correlation sudden change occurs at certain time. For a complete view of this interesting change, we show the whole evolution of mutual information, classical correlation and quantum correlation with no interactions between the qubits in Fig.2, The mutual information goes down monotonically as the system evolves. However, it is shown clearly that there are several crosses and sudden changes of the classical and quantum correlation. At first the quantum correlation shows a high speed of decay as a result of the interaction with the bath. Then the decay stops and the quantum correlation even shows a little increase, which clearly demonstrate the non-Markovian memory effects. However, the 
classical decoherence speeds up and at around $t=1.7 / \delta$ these two correlations reach the same value. A cross immediately after the time shows the lager discord than the classical correlation during a short interval. Another cross emerges at $t \approx 3.6 / \delta$. After this we may not find any crosses and the quantum correlation is then always larger than classical correlation. With careful observation we know that at both crosses and some other time points (such as $t \approx 6.2 / \delta$ and $t \approx 8.4 / \delta$ ) there are sudden changes of the correlations. These sudden changes catch interests because they may be results of some quantum phase transitions or the transition of classical and quantum decoherence [12]. We also find that the length of the intervals between these changes are all nearly $2.2 / \delta$. This phenomenon may indicate some mechanisms of the system evolution in a common non-Markovian environment.

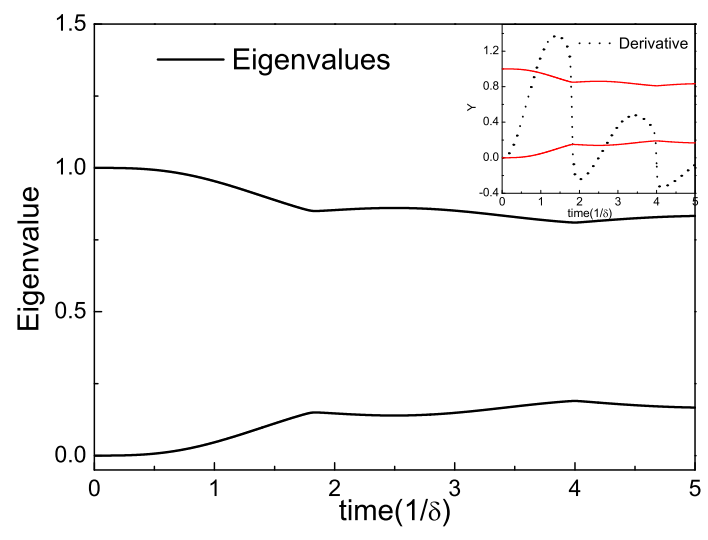

FIG. 3: (color online) The eigenvalues of the density operator $\rho_{A}^{j}$ after the maximization procedure as a function of time. The dotted line in the inserted figure at the top right corner is the derivative of the lower eigenvalue.

In order to investigate the sudden changes, we calculate the eigenvalues of $\rho_{A}^{j}$ after the maximization procedure in Eq.4. Fig. 3 3 shows the first two sudden changes of the eigenvalues. These two curves have exactly the opposite trends and both have two inflectional points, at which the derivative can become quite sharp, as we show in the inserted figure. Obviously the two points correspond to the sudden changes in the classical and quantum correlations.

Next we concentrate on the influence of coupling strength. We choose $\zeta=0.5 \delta$ and $\zeta=0.1 \delta$ for comparison which are shown in Fig.4 The left figure shows a quite common dynamics of the correlations, with classical correlation always larger than quantum counterpart. When the strength of the interaction between the two qubits decreases to $0.1 \delta$, crosses 

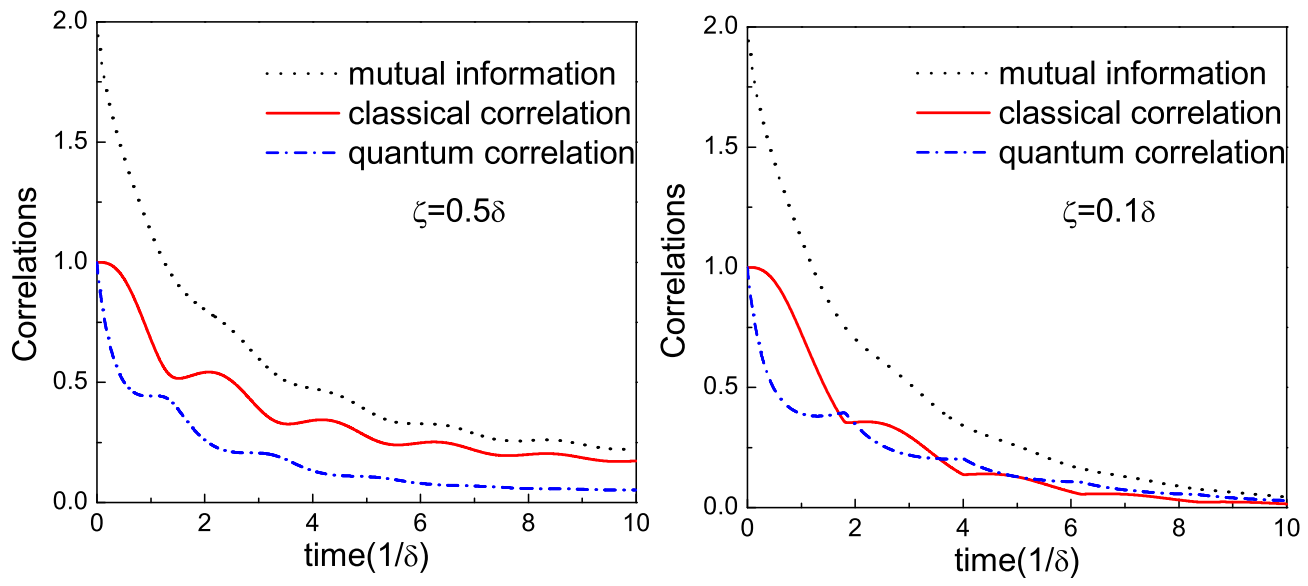

FIG. 4: (color online) Mutual information, classical correlation and quantum correlation for different coupling strength between the qubits. $\zeta=0.5 \delta$ in the left figure and $\zeta=0.1 \delta$ in the right one.

begin to emerge as the quantum correlation rises and classical correlation goes down. However, with careful observation we find that the changes are not so sharp as the ones in Fig.1 where $\zeta=0$. So we can infer that at the point of $\zeta=0$ some interesting phenomenon may happen such as the correlation sudden changes.

Apart from the effects of the interactions between the two qubits, the initial states of the system also play an important role on the evolution of the correlations, as shown in Fig.5. We change the initial state from odd $(|\psi\rangle=1 / \sqrt{2}(|10\rangle-|01\rangle))$ to even parity $(|\psi\rangle=1 / \sqrt{2}(|11\rangle-|00\rangle))$. The parameters are chosen to be the same as those in Fig.2. Sudden transitions between quantum and classical correlations can be found which has been demonstrated in other systems [11, 12]. This kind of transition can occur several times in our system during the evolution such as at the time $t=1 / \delta, t=2 / \delta$ and so on. 


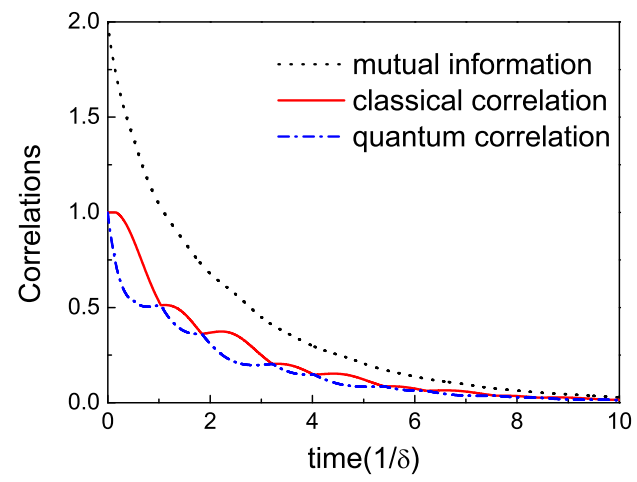

FIG. 5: (color online) The black dotted, red solid and blue dash-dotted lines represent the mutual information, classical correlation and quantum correlation as functions of time, respectively, with an initial state of $|\psi\rangle=1 / \sqrt{2}(|11\rangle-|00\rangle)$.

\section{CONCLUSION}

We study the evolution of the classical and quantum correlations of a two-qubit system in independent non-Markovian environments, applying the newly developed hierarchy equations approach. The influence of initial state and coupling strength between the qubits are investigated. Crosses and sudden changes of classical and quantum correlations are illustrated. We further show sudden transition between classical and quantum correlations for certain initial state.

\section{ACKNOWLEDGEMENT}

This work was supported by the National Fundamental Research Program and National Natural Science Foundation of China (Grant Nos. 60921091, 10874162 and 10734060).

[1] F. F. Fanchini, T. Werlang, C. A. Brasil, L. G. E. Arruda, and A. O. Caldeira, Phys. Rev. A. 81, 052107 (2010).

[2] J. Maziero, L. C. Céleri, R. M. Serra, and V. Vedral, Phys. Rev. A. 80, 044102 (2009).

[3] H. T. Wang, C. F. Li, Y. Zou, R. C. Ge, and G. C. Guo, arXiv:1010.3402,

[4] A. G. Dijkstra and Y. Tanimura, Phys. Rev. Lett. 104, 250401 (2010). 
[5] B. Groisman, S. Popescu, and A. Winter, Phys. Rev. A. 72, 032317 (2005).

[6] H. Ollivier and W. H. Zurek, Phys. Rev. Lett. 88, 017901 (2001).

[7] V. Vedral, The elusive source of quantum ef fectiveness.

[8] V. Vedral, Rev. Mod. Phys. 74, 197 (2002).

[9] L. Henderson and V. Vedral, J. Phys. A: Math. Gen. 34, 6899 (2001).

[10] J. S. Xu, C. F. Li, C. J. Zhang, X. Y. Xu, Y. S. Zhang, and G. C. Guo, Phys. Rev. A. 82, $042328(2010)$.

[11] J. S. Xu, X. Y. Xu, C. F. Li, C. J. Zhang, X. B. Zou, and G. C. Guo, Nat. Commun 1, 7 (2010).

[12] L. Mazzola, J. Piilo, and S. Maniscalco, Phys. Rev. Lett. 104, 200401 (2001).

[13] A. Ishizaki and Y. Tanimura, J. Phys. Soc. Jpn. 74, 3131 (2005).

[14] A. J. Leggett, S. Chakravarty, A. T. Dorsey, M. P. A. Fisher, A. Garg and W. Zwerger, Rev. Mod. Phys. 59, 1 (1987).

[15] M. Ali, A. R. P. Rau, and G. Alber, Phys. Rev. A. 81, 042105 (2010). 